\title{
Hidden variables as computational tools: the construction of a relativistic spinor field"
}

\author{
Peter Holland \\ Green College \\ University of Oxford \\ Oxford OX2 6HG \\ England \\ peter.holland@green.ox.ac.uk
}

18 January 2005

\begin{abstract}
Hidden variables are usually presented as potential completions of the quantum description. We describe an alternative role for these entities, as aids to calculation in quantum mechanics. This is illustrated by the computation of the time-dependence of a massless relativistic spinor field obeying Weyl's equation from a single-valued continuum of deterministic trajectories (the "hidden variables"). This is achieved by generalizing the exact method of state construction proposed previously for spin 0 systems to a general Riemannian manifold from which the spinor construction is extracted as a special case. The trajectories form a noncovariant structure and the Lorentz covariance of the spinor field theory emerges as a kind of collective effect. The method makes manifest the spin 1/2 analogue of the quantum potential that is tacit in Weyl's equation. This implies a novel definition of the "classical limit" of Weyl's equation.
\end{abstract}

KEY WORDS: hidden variables; spinor; quantum hydrodynamic trajectories; Weyl's equation; Riemannian geometry; classical limit; Lorentz covariance.

PACS: 03.65.Ta.

\section{INTRODUCTION}

Broadly speaking, hidden variables are quantities that complete the quantum description by determining, in conjunction with the state vector, the outcomes of all possible measurements (with their attendant distributions) that are relevant to the domain of the quantum theory considered $^{(1,2)}$. The historical discussion surrounding hidden variables has tended to concentrate on their existence and properties as theoretical entities underpinning the quantum formalism and their benefits are perceived primarily in terms of the insight they provide into quantum reality. Less consideration has been given to the possible practical value of hidden variables in solving technical problems in quantum mechanics. For example, one may enquire whether these variables could assist in solving the Schrödinger equation in a way analogous

\footnotetext{
* Article written in honour of Emilio Santos.
} 
to, say, the computation of thermodynamic relations from microstates in statistical mechanics. Apart from the potential benefits to quantum mechanics, this could prove a fruitful avenue of investigation within the hidden-variable programme for, should the additional variables play such an efficacious role, this may provide alternative evidence of their consistency with the quantum formalism. Indeed, such an approach may provide the basis of an alternative picture of quantum mechanics in the technical sense of transformation theory.

In this article we shall describe such a possibility. Specifically, we present an exact scheme to calculate the time-dependent wavefunction for a massless relativistic spin $1 / 2$ system obeying Weyl's equation from a single-valued continuum of deterministic trajectories (the (ensemble of) "hidden variables"). This extends the method described recently for spin $0^{(3)}$ and spin $1^{(4)}$ systems.

In the previous papers it was argued that a natural language for the theory is offered by the hydrodynamic analogy, in which wave mechanics corresponds to the Eulerian picture and the trajectory theory to the Lagrangian picture. The Lagrangian model for the quantum fluid may be developed from a variational principle, and the Euler-Lagrange equations imply a fourth-order nonlinear partial differential equation to calculate the trajectories of the fluid particles as functions of their initial coordinates and the initial wavefunction. Restricting the admissible solutions to those consistent with quasi-potential flow, the wavefunction is computed via the standard map between the Lagrangian coordinates and the Eulerian fields. The key step in the computation is to express the initial coordinates as functions of space and time. The map supplies the analogue in this model of Huygens' principle in wave mechanics, the effect of the superposition principle being represented by a nonclassical force on each fluid element. The theory provides a picture of quantum processes complementary to wave mechanics, and shows that the deterministic trajectory concept may be regarded as a basic component of the quantum description (and potentially of other wave theories) and not just of one of its interpretations (e.g., the de Broglie-Bohm model). In order to include spin, the hydrodynamic model is extended so that the fluid particles acquire internal rotational freedoms in addition to translational ones.

An Eulerian hydrodynamic picture for Weyl's equation has been developed previously $^{(5)}$, along the lines of earlier methods used for spin $1 / 2$ systems (for references see $\left.{ }^{(6)}\right)$. However, in connection with our programme, it is not clear how to develop a suitable Lagrangian-coordinate version of the standard hydrodynamic model. A natural definition of the flow lines would be the integral curves of the space components of the current divided by the density. It appears that an ensemble of such paths would not contain sufficient information to construct the four real field components in the 2-spinor according to the method set out in (3)

This problem may be circumvented by noting that the standard approach works with a (angular momentum) representation of the quantum theory in which the rotational freedoms appear as discrete indices in the wavefunction ${ }^{(6)}$. The local fluid quantities (density, velocity, spin vector,...) are defined by "averaging" over these indices, which implies a loss of information and reproducing through them all the information in the wavefunction requires introducing ever more complex quantities and combinations of quantities. The alternative procedure advocated in ${ }^{(6)}$ and used here is to start from the angular coordinate representation in which the spin freedoms are represented as continuous parameters $\alpha$ (Euler angles) in the wavefunction, on the same footing as the spatial variables $x: \psi(x, \alpha, t)$. This implies a physically clearer and simpler hydrodynamic-like model, in both its Eulerian and Lagrangian guises. The phase $S$ of the wavefunction is immediately identifiable and the equations for the fluid paths are defined in terms of the gradients of $S$ with respect to the coordinates, obvious generalizations of the spin 0 theory. The approach also provides a natural framework to study vortices, and it has the merit of locating spin analogues of the spin 0 quantum potential, as we shall see. 
The final advantage of the angular coordinate approach is that it allows us to extend our method of wavefunction construction to the spin $1 / 2$ field. This is achieved by generalizing the theory of ${ }^{(3)}$ to a general Riemannian manifold from which the spinor construction is extracted as a special case. The Lagrangian-coordinate model presented here is not relativistically covariant and we shall see that the Lorentz covariance of the (Eulerian) field theory can be derived from the non-covariant theory as a kind of collective effect.

\section{HYDRODYNAMIC FORMULATION OF WEYL'S EQUATION}

\subsection{Continuous representation of Weyl's equation}

It turns out that the massless spin 1/2 theory may be developed applying the method used for the massless spin 1 case $^{(4)}$ with only minor changes. Weyl's equation in Schrödinger form is

$$
i \hbar \frac{\partial \chi_{a}}{\partial t}=-i \hbar c\left(\sigma_{i}\right)_{a b} \partial_{i} \chi_{b}
$$

where $\chi_{a}$ is a 2-component spinor, $a, b=1,2$, and

$$
\left(\sigma_{1}\right)_{a b}=\left(\begin{array}{cc}
0 & 1 \\
1 & 0
\end{array}\right), \quad\left(\sigma_{2}\right)_{a b}=\left(\begin{array}{cc}
0 & -i \\
i & 0
\end{array}\right), \quad\left(\sigma_{3}\right)_{a b}=\left(\begin{array}{cc}
1 & 0 \\
0 & -1
\end{array}\right),
$$

are the Pauli matrices. Summation over repeated indices is always assumed. To pass to the angular coordinate representation, we use the definition of the Euler angles $\left(\alpha_{r}\right)=(\alpha, \beta, \gamma), r$ $=1,2,3$, and conventions of ${ }^{(6)}$. The angular momentum components become differential operators:

$$
\left.\begin{array}{l}
\hat{M}_{1}=i \hbar\left(\cos \beta \partial_{\alpha}-\sin \beta \cot \alpha \partial_{\beta}+\sin \beta \operatorname{cosec} \alpha \partial_{\gamma}\right) \\
\hat{M}_{2}=i \hbar\left(-\sin \beta \partial_{\alpha}-\cos \beta \cot \alpha \partial_{\beta}+\cos \beta \operatorname{cosec} \alpha \partial_{\gamma}\right) \\
\hat{M}_{3}=i \hbar \partial_{\beta}
\end{array}\right\}
$$

where $\partial_{\alpha}=\partial / \partial \alpha$ etc. Weyl's equation (1) becomes

$$
i \hbar \frac{\partial \psi(x, \alpha)}{\partial t}=-2 i c \hat{M}_{i} \partial_{i} \psi(x, \alpha)
$$

or, introducing the real operator $\hat{\lambda}_{i}=\hat{M}_{i} /(-i \hbar)$,

$$
i \hbar \frac{\partial \psi}{\partial t}=-2 c \hbar \hat{\lambda}_{i} \partial_{i} \psi
$$

where $\psi$ is a function on the manifold $M=\mathfrak{R}^{3} \otimes S U(2)$ whose points are labelled by $(x, \alpha)$. In this representation the wavefunction may be expanded in terms of an orthonormal set of spin $1 / 2$ basis functions $u_{a}(\alpha)^{(6)}$ :

$$
\psi(x, \alpha, t)=\chi_{a}(x, t) u_{a}(\alpha), \quad a=1,2,
$$


where

$$
u_{1}(\alpha)=(2 \sqrt{2} \pi)^{-1} \cos (\alpha / 2) e^{-i(\beta+\gamma) / 2}, \quad u_{2}(\alpha)=-i(2 \sqrt{2} \pi)^{-1} \sin (\alpha / 2) e^{i(\beta-\gamma) / 2},
$$

with

$$
\int u_{a}^{*}(\alpha) u_{b}(\alpha) d \Omega=\delta_{a b}, \quad d \Omega=\sin \alpha d \alpha d \beta d \gamma, \quad \alpha \in[0, \pi], \beta \in[0,2 \pi], \gamma \in[0,4 \pi]
$$

It is readily checked that

$$
\int u_{a}^{*}(\alpha) \hat{M}_{i} u_{b}(\alpha) d \Omega=(\hbar / 2)\left(\sigma_{i}\right)_{a b}
$$

and multiplying (4) by $u_{a}^{*}(\alpha)$ and using (8) we recover (1).

In this formalism the field equation (4) comes out as a second-order partial differential equation, and summation over $a$ is replaced by integration over $\alpha_{r}$. For example, for the components of the 4-current $c \chi_{a}^{*}\left(\sigma_{\mu}\right)_{a b} \chi_{b}$ we have the alternate expressions

$$
\begin{aligned}
& c \chi_{a}^{*} \chi_{a}=c \int|\psi(x, \alpha)|^{2} d \Omega \\
& c \chi_{a}^{*}\left(\sigma_{i}\right)_{a b} \chi_{b}=\frac{2 c}{\hbar} \int \psi^{*}(x, \alpha) \hat{M}_{i} \psi(x, \alpha) d \Omega
\end{aligned}
$$

To obtain the hydrodynamic model we follow Madelung ${ }^{(7)}$ and express the wavefunction in polar form: $\psi=\sqrt{\rho} \exp (i S / \hbar)$. Splitting the wave equation (5) into real and imaginary parts then gives the relations

$$
\begin{aligned}
& \frac{\partial S}{\partial t}+\frac{2 c}{\hbar} \hat{\lambda}_{i} S \partial_{i} S+Q=0 \\
& \frac{\partial \rho}{\partial t}+\frac{2 c}{\hbar} \partial_{i}\left(\rho \hat{\lambda}_{i} S\right)+\frac{2 c}{\hbar} \hat{\lambda}_{i}\left(\rho \partial_{i} S\right)=0
\end{aligned}
$$

where

$$
Q=-2 c \hbar \frac{\hat{\lambda}_{i} \partial_{i} \sqrt{\rho}}{\sqrt{\rho}} .
$$

These two equations are equivalent to Weyl's equation (1), subject to the proviso that $\rho$ and $S$ obey certain conditions inherited from $\psi$. In particular, the single-valuedness of the wavefunction requires

$$
\oint_{C_{0}} \partial_{i} S d x_{i}+\partial_{r} S d \alpha_{r}=n h, \quad n \in Z,
$$

where $C_{0}$ is a closed curve in $M$. In the hydrodynamic model the number $|n|$ is interpreted as the net strength of the vortices contained in $C$. These occur in nodal regions $(\psi=0)$ where $S$ is singular. 
Comparing (13) with the Eulerian continuity equation corresponding to a fluid of density $\rho$ with translational and rotational freedoms,

$$
\frac{\partial \rho}{\partial t}+\partial_{i}\left(\rho v_{i}\right)+\hat{\lambda}_{i}\left(\rho \omega_{i}\right)=0
$$

we shall make the following identifications for the velocity and angular velocity fields ${ }^{1}$ :

$$
v_{i}=(2 c / \hbar) \hat{\lambda}_{i} S, \quad \omega_{i}=(2 c / \hbar) \partial_{i} S .
$$

Clearly, we obtain a kind of potential flow (strictly, quasi-potential in view of (15)), the potential being $(2 c / \hbar) S$. Note, however, the unorthodox connections between the potential and the hydrodynamic quantities $-v_{i}\left(\omega_{i}\right)$ is a gradient with respect to the angular (spatial) variables. The quantity $Q$ in (12) is the analogue for Weyl's equation of the quantum potential that appears in the polar decomposition of the Schrödinger equation for a massive particle. As we shall see in Sec. 4, $Q$ has the classic form " $-\nabla^{2} \sqrt{\rho} / \sqrt{\rho}$ " when the appropriate metric on $M$ is identified.

From the Bernoulli-like (or Hamilton-Jacobi-like) equation (12) we may obtain the analogue(s) of Euler's force law for the spin 1/2 fluid. Applying first $\partial_{i}$, rearranging and using (17) we get

$$
\left(\frac{\partial}{\partial t}+v_{j} \partial_{j}+\omega_{j} \hat{\lambda}_{j}\right) \omega_{i}=-\frac{2 c}{\hbar} \partial_{i} Q
$$

Next, applying $\hat{\lambda}_{i}$ and using $\left[\hat{\lambda}_{i}, \hat{\lambda}_{j}\right]=-\varepsilon_{i j k} \hat{\lambda}_{k}$ gives

$$
\left(\frac{\partial}{\partial t}+v_{j} \partial_{j}+\omega_{j} \hat{\lambda}_{j}\right) v_{i}=\varepsilon_{i j k} \omega_{j} v_{k}-\frac{2 c}{\hbar} \hat{\lambda}_{i} Q
$$

which contains a precession-type term in addition to the quantum contribution.

An alternative representation of the internal angular motion is in terms of the velocity fields $v_{r}(x, \alpha, t)$ conjugate to the Euler angles. These are connected to the components of the vector angular velocity field by the relations

$$
\omega_{i}=\left(A^{-1}\right)_{i r} v_{r}, \quad v_{r}=A_{r i} \omega_{i}, \quad i, r=1,2,3,
$$

where

$$
A_{i r}=\left(\begin{array}{ccc}
-\cos \beta & \sin \beta \cot \alpha & -\sin \beta \operatorname{cosec} \alpha \\
\sin \beta & \cos \beta \cot \alpha & -\cos \beta \operatorname{cosec} \alpha \\
0 & -1 & 0
\end{array}\right), \quad\left(A^{-1}\right)_{i r}=\left(\begin{array}{ccc}
-\cos \beta & 0 & -\sin \alpha \sin \beta \\
\sin \beta & 0 & -\sin \alpha \cos \beta \\
0 & -1 & -\cos \alpha
\end{array}\right) .
$$

Relations (3) may be written $\hat{\lambda}_{i}=A_{i r} \partial_{r}$ and it is easy to show using the result $\left(A^{-1}\right)_{i r} A_{i s}=\delta_{r s}$ that $\omega_{j} \hat{\lambda}_{j}=v_{r} \partial_{r}$. In terms of the conjugate velocities Euler's equations (18) and (19) become, on substituting (20),

\footnotetext{
${ }^{1}$ The uniqueness of this identification needs careful discussion. For examination of some of the issues involved see ${ }^{(8,9)}$.
} 


$$
\begin{aligned}
& \left(\frac{\partial}{\partial t}+v_{j} \partial_{j}+v_{r} \partial_{r}\right) v_{s}+A_{s i} \partial_{r}\left(A^{-1}\right)_{i q} v_{q} v_{r}=-\frac{2 c}{\hbar} A_{s i} \partial_{i} Q, \quad q, r, s=1,2,3, \\
& \left(\frac{\partial}{\partial t}+v_{j} \partial_{j}+v_{r} \partial_{r}\right) v_{i}+\varepsilon_{i j k}\left(A^{-1}\right)_{k r} v_{j} v_{r}=-\frac{2 c}{\hbar} \hat{\lambda}_{i} Q .
\end{aligned}
$$

We shall see in Sec. 4 that the last terms on the left-hand sides of these equations may be attributed a geometrical interpretation.

\subsection{Fluid paths}

The paths $x=x\left(x_{0}, \alpha_{0}, t\right), \alpha=\alpha\left(x_{0}, \alpha_{0}, t\right)$ of the fluid particles in $M$ are obtained from the Eulerian velocity functions by solving the differential equations

$$
v_{i}(x, \alpha, t)=\frac{\partial x_{i}}{\partial t}, \quad v_{r}(x, \alpha, t)=\frac{\partial \alpha_{r}}{\partial t} .
$$

Combining these formulas with (17) and (20) we have

$$
\frac{\partial x_{i}}{\partial t}=\frac{2 c}{\hbar} A_{i r} \partial_{r} S, \quad \frac{\partial \alpha_{r}}{\partial t}=\frac{2 c}{\hbar} A_{r i} \partial_{i} S, \quad i, r=1,2,3,
$$

where we substitute $x=x\left(x_{0}, \alpha_{0}, t\right), \alpha=\alpha\left(x_{0}, \alpha_{0}, t\right)$ on the right-hand sides. These relations generally imply a complex coupling between the translational and angular freedoms. Note that the connection between the fields and the paths embodied in (24) breaks relativistic covariance, for this is not a Lorentz covariant system of equations (e.g., $v_{i}$ is not a Lorentz 3vector (i.e., $\equiv u^{i} / u^{0}$ where $u^{A}$ is a 4 -vector)).

\subsection{Classical limit}

There are circumstances where $Q$ is both sufficiently small numerically and slowly varying with respect to the (six) coordinates that it and its gradients may be neglected in (12), (18) and (19). We shall term this situation the "classical limit" of Weyl's equation. The motion of a fluid particle in this case can be expressed as a geodesic for a suitably chosen metric (see (35)). According to (18) and (19) along a fluid path the angular velocity is constant and the translational velocity is either constant or precesses about the angular velocity vector. Thus, the limiting spatial flow is composed of linear or helical orbits. It may be shown that the classical model as defined here can be "quantized" according to the usual rules to get back the massless wave equation ${ }^{(10)}$.

\subsection{The nonzero mass case}

In the continuous representation, the Dirac equation for a massive spin $1 / 2$ particle may be expressed as ${ }^{(11)}$

$i \hbar \frac{\partial \phi(x, \alpha)}{\partial t}=-\frac{4 i c}{\hbar} \hat{M}_{1}^{\prime} \hat{M}_{i} \partial_{i} \phi(x, \alpha)+\frac{2 m c^{2}}{\hbar} \hat{M}_{3}^{\prime} \phi(x, \alpha)$ 
where the operators $\hat{M}_{i}^{\prime}$ obey the anomalous angular momentum commutation relations. This is a third-order differential equation and the trajectory technique for computing the Weyl spinor we describe below does not extend readily to this case. We shall discuss this problem elsewhere.

\section{LAGRANGIAN-COORDINATE CONSTRUCTION OF THE WAVEFUNCTION IN A RIEMANNIAN MANIFOLD}

\subsection{Newton's law for a fluid element}

We here review the method of constructing the wavefunction from hydrodynamic trajectories in arbitrary coordinates $x^{\mu}$ in an $N$-dimensional Riemannian manifold $M$ with (static) metric $g_{\mu \nu}(x), \mu, \nu, \ldots=1, \ldots, N .^{(4)}$ In this space, the history of the fluid is encoded in the positions $\xi\left(\xi_{0}, t\right)$ of the distinct fluid elements at time $t$, each particle being distinguished by its position $\xi_{0}$ at $t=0$. We assume that the mapping between these two sets of coordinates is singlevalued and differentiable with respect to $\xi_{0}$ and $t$ to whatever order is necessary, and that the inverse mapping $\xi_{0}(\xi, t)$ exists and has the same properties.

Let $P_{0}\left(\xi_{0}\right)$ be the initial density of some continuously distributed quantity in $M$ and $g=\operatorname{det} g_{\mu v}$. Then the quantity in an elementary volume $d^{N} \xi_{0}$ attached to the point $\xi_{0}$ is given by $P_{0}\left(\xi_{0}\right) \sqrt{-g\left(\xi_{0}\right)} d^{N} \xi_{0}$. The conservation of this quantity in the course of the motion of the fluid element is expressed through the relation

$$
P\left(\xi\left(\xi_{0}, t\right)\right) \sqrt{-g\left(\xi\left(\xi_{0}, t\right)\right)} d^{N} \xi\left(\xi_{0}, t\right)=P_{0}\left(\xi_{0}\right) \sqrt{-g\left(\xi_{0}\right)} d^{N} \xi_{0}
$$

or

$$
P\left(\xi_{0}, t\right)=D^{-1}\left(\xi_{0}, t\right) P_{0}\left(\xi_{0}\right)
$$

where

$$
D\left(\xi_{0}, t\right)=\sqrt{g(\xi) / g\left(\xi_{0}\right)} J\left(\xi_{0}, t\right), \quad 0<D<\infty
$$

and $J$ is the Jacobian of the transformation between the two sets of coordinates:

$$
J=\frac{1}{N !} \varepsilon_{\mu_{1} \ldots \mu_{N}} \varepsilon^{\nu_{1} \ldots v_{N}} \frac{\partial \xi^{\mu_{1}}}{\partial \xi_{0}^{v_{1}}} \ldots \frac{\partial \xi^{\mu_{N}}}{\partial \xi_{0}^{v_{N}}}
$$

We assume that the Lagrangian for the set of fluid particles comprises a kinetic term and an internal potential that represents a certain kind of particle interaction:

$$
L=\int P_{0}\left(\xi_{0}\right)\left(\frac{1}{2} g_{\mu \nu}(\xi) \frac{\partial \xi^{\mu}}{\partial t} \frac{\partial \xi^{v}}{\partial t}-\frac{1}{2} c^{2} l^{2} g^{\mu \nu}(\xi) \frac{1}{P^{2}} \frac{\partial P}{\partial \xi^{\mu}} \frac{\partial P}{\partial \xi^{v}}\right) \sqrt{-g\left(\xi_{0}\right)} d^{N} \xi_{0}
$$

Here $P_{0}$ and $g_{\mu v}$ are prescribed functions, $\xi=\xi\left(\xi_{0}, t\right), l$ is a constant with the dimension of length, and we substitute for $P$ from (28) and write 


$$
\frac{\partial}{\partial \xi^{\mu}}=J^{-1} J_{\mu}^{v} \frac{\partial}{\partial \xi_{0}^{v}}
$$

where

$$
J_{\mu}^{v}=\frac{\partial J}{\partial\left(\partial \xi^{\mu} / \partial \xi_{0}^{v}\right)}
$$

is the cofactor of $\partial \xi^{\mu} / \partial \xi_{0}^{v}$. The latter satisfies

$$
\frac{\partial \xi^{\mu}}{\partial \xi_{0}^{v}} J_{\mu}^{\sigma}=J \delta_{v}^{\sigma}
$$

It is assumed that $P_{0}$ and its derivatives vanish at infinity, which ensures that the surface terms in the variational principle vanish. It is remarkable that the internal potential in (31) applies to both systems with and without spin, the cases being distinguished by the choice of coordinates and the functional dependences of the metric and initial density.

Varying the coordinates, the Euler-Lagrange equations of motion for the $\xi_{0}$ th fluid particle moving in the "field" of the other particles take the form of Newton's second law in general coordinates:

$$
\frac{\partial^{2} \xi^{\mu}}{\partial t^{2}}+\left\{\begin{array}{c}
\mu \\
v \sigma
\end{array}\right\} \frac{\partial \xi^{v}}{\partial t} \frac{\partial \xi^{\sigma}}{\partial t}=-\frac{2 c l}{\hbar} g^{\mu \nu} \frac{\partial Q}{\partial \xi^{v}}
$$

where $\left\{\begin{array}{l}\mu \\ v \sigma\end{array}\right\}=\frac{1}{2} g^{\mu \rho}\left(\partial g_{\sigma \rho} / \partial \xi^{v}+\partial g_{v \rho} / \partial \xi^{\sigma}-\partial g_{v \sigma} / \partial \xi^{\rho}\right)$ and

$$
Q=\frac{-\hbar c l}{\sqrt{-g P}} \frac{\partial}{\partial \xi^{\mu}}\left(\sqrt{-g} g^{\mu \nu} \frac{\partial \sqrt{P}}{\partial \xi^{v}}\right)
$$

We have written the force term on the right-hand side of (35) in condensed form and substituting for $P$ from (28) and for the derivatives with respect to $\xi$ from (32) we obtain a highly complex fourth order (in $\xi_{0}$ ) local nonlinear partial differential equation. We shall see that from the solutions $\xi=\xi\left(\xi_{0}, t\right)$, subject to specification of $\partial \xi_{0}^{\mu} / \partial t$ whose determination is discussed next, we may derive solutions to Schrödinger's equation.

\subsection{Quasi-potential flow}

To obtain a flow that is representative of Schrödinger evolution we need to restrict the initial conditions of (35) to those that correspond to what we term "quasi-potential" flow. This means that the initial covariant components of the velocity field are of the form (we introduce the factor $2 c l / \hbar$ with an eye to the spin application)

$$
g_{\mu v}\left(\xi_{0}\right) \frac{\partial \xi_{0}^{\mu}}{\partial t}=\frac{2 c l}{\hbar} \frac{\partial S_{0}\left(\xi_{0}\right)}{\partial \xi_{0}^{v}}
$$

but the flow is not irrotational everywhere because the potential $S_{0}\left(\xi_{0}\right)$ obeys the quantization condition 


$$
\oint_{C} \frac{\partial S_{0}\left(\xi_{0}\right)}{\partial \xi_{0}^{\mu}} d \xi_{0}^{\mu}=n h, \quad n \in Z
$$

where $C$ is a closed curve composed of fluid particles. The requirement (38) evidently restricts the circulation of the covariant components of the initial velocity (37). If it exists, the vorticity occurs in nodal regions (where the density vanishes) and it is assumed that $C$ passes through a region of "good" fluid, where $P_{0} \neq 0$. It can be shown that the quasi-potential property is preserved by the dynamical equation (35). That is, at time $t$, we have

$$
g_{\mu \nu} \frac{\partial \xi^{v}}{\partial t}=\frac{2 c l}{\hbar} \frac{\partial S}{\partial \xi^{\mu}}, \quad S\left(\xi_{0}(\xi, t), t\right)=S_{0}+\int_{0}^{t}\left(\frac{\hbar}{4 c l} g_{\mu \nu} \frac{\partial \xi^{\mu}}{\partial t} \frac{\partial \xi^{v}}{\partial t}-Q\right) d t .
$$

and

$$
\frac{\partial}{\partial t} \oint_{C(t)} g_{\mu v} \frac{\partial \xi^{v}}{\partial t} d \xi^{\mu}=0
$$

where $C(t)$ is the evolute of the fluid particles that compose $C$. We conclude that each particle retains forever the quasi-potential property if it possesses it at any moment.

\subsection{Derivation of Schrödinger's equation}

The fundamental link between the particle (Lagrangian) and wave-mechanical (Eulerian) pictures is defined by the following expression for the Eulerian density:

$$
P(x, t) \sqrt{-g(x)}=\int \delta\left(x-\xi\left(\xi_{0}, t\right)\right) P_{0}\left(\xi_{0}\right) \sqrt{-g\left(\xi_{0}\right)} d^{N} \xi_{0} .
$$

The corresponding formula for the Eulerian velocity is contained in the expression for the current:

$$
P(x, t) \sqrt{-g(x)} v^{\mu}(x, t)=\int \frac{\partial \xi^{\mu}\left(\xi_{0}, t\right)}{\partial t} \delta\left(x-\xi\left(\xi_{0}, t\right)\right) P_{0}\left(\xi_{0}\right) \sqrt{-g\left(\xi_{0}\right)} d^{N} \xi_{0}
$$

Evaluating the integrals, (41) and (42) are equivalent to the following local expressions

$$
\begin{aligned}
& P(x, t) \sqrt{-g(x)}=\left.J^{-1}\right|_{\xi_{0}(x, t)} P_{0}\left(\xi_{0}(x, t)\right) \sqrt{-g\left(\xi_{0}(x, t)\right)} \\
& v^{\mu}(x, t)=\left.\frac{\partial \xi^{\mu}\left(\xi_{0}, t\right)}{\partial t}\right|_{\xi_{0}(x, t)} .
\end{aligned}
$$

These formulas enable us to translate the Lagrangian flow equations into Eulerian language. Differentiating (41) with respect to $t$ and using (42) we deduce the continuity equation

$$
\frac{\partial P}{\partial t}+\frac{1}{\sqrt{-g}} \frac{\partial}{\partial x^{\mu}}\left(P \sqrt{-g} v^{\mu}\right)=0
$$


Next, differentiating (42) and using (35) and (45) we get the analogue of Euler's classical equation:

$$
\frac{\partial v^{\mu}}{\partial t}+v^{v} \frac{\partial v^{\mu}}{\partial x^{v}}+\left\{\begin{array}{c}
\mu \\
v \sigma
\end{array}\right\} v^{v} v^{\sigma}=-\frac{2 c l}{\hbar} g^{\mu \nu} \frac{\partial Q}{\partial x^{v}},
$$

where $Q$ is given by (36) with $\xi$ replaced by $x$. Finally, the quasi-potential condition (39) becomes

$$
v^{\mu}=\frac{2 c l}{\hbar} g^{\mu \nu} \frac{\partial S(x, t)}{\partial x^{v}}
$$

Formulas (43) and (44) give the general solution of the coupled continuity and Euler equations (45) and (46) in terms of the paths and initial density.

To establish the connection between the Eulerian equations and Schrödinger's equation we note that, using (47), (46) can be written

$$
\frac{\partial}{\partial x^{\mu}}\left(\frac{\partial S}{\partial t}+\frac{c l}{\hbar} g^{v \sigma} \frac{\partial S}{\partial x^{v}} \frac{\partial S}{\partial x^{\sigma}}+Q\right)=0 .
$$

The quantity in brackets is thus a function of time. Since the addition of a function of time to $S$ does not affect the velocity field, we may absorb the function in $S$, i.e., set it to zero. Then

$$
\frac{\partial S}{\partial t}+\frac{c l}{\hbar} g^{v \sigma} \frac{\partial S}{\partial x^{v}} \frac{\partial S}{\partial x^{\sigma}}+Q=0
$$

Combining (49) with (45) (where we substitute (47)) we find that the function $\psi(x, t)=\sqrt{P} \exp (i S / \hbar)$ obeys the free Schrödinger equation in general coordinates:

$$
i \hbar \frac{\partial \psi}{\partial t}=\frac{-\hbar c l}{\sqrt{-g}} \frac{\partial}{\partial x^{\mu}}\left(\sqrt{-g} g^{\mu \nu} \frac{\partial \psi}{\partial x^{v}}\right)
$$

(for a system of "mass" $\hbar / 2 c l$ ). We have deduced the wave equation from the collective particle motion obeying the Lagrangian path equation (35) subject to the quasi-potential requirement. The quantization condition (40) becomes here

$$
\oint_{C_{0}} \frac{\partial S(x, t)}{\partial x^{\mu}} d x^{\mu}=n h, \quad n \in Z
$$

where $C_{0}$ is a closed curve fixed in space that does not pass through nodes. This is a consistent subsidiary condition on solutions since it is easy to see using (48) that the value of (51) is preserved in time as long as nodes do not cross $C_{0}$.

\section{CONSTRUCTION OF THE SPINOR FIELD FROM THE TRAJECTORIES}

We specialize the treatment of the last section to the manifold $M=\Re^{3} \otimes S U(2)$ with coordinates $x^{\mu}=\left(x_{i}, \alpha_{r}\right)$, metric 


$$
g^{\mu v}=\left(\begin{array}{cc}
0 & l^{-1} A_{i r} \\
l^{-1} A_{r i} & 0
\end{array}\right), \quad g_{\mu \nu}=\left(\begin{array}{cc}
0 & l\left(A^{-1}\right)_{i r} \\
l\left(A^{-1}\right)_{r i} & 0
\end{array}\right), \quad i, r=1,2,3,
$$

where $A_{i r}$ is given by (21), and density $P=\rho / l^{3}$. Using, in particular, the results $\partial_{r}\left(\sqrt{-g} g^{i r}\right)=0$ and $l \mathrm{~g}^{i r} \partial_{r}=\hat{\lambda}_{i}$, and inserting the latter in the relations $\left[\hat{\lambda}_{i}, \hat{\lambda}_{j}\right]=-\varepsilon_{i j k} \hat{\lambda}_{k}$ which gives $g^{i r}\left(\partial_{s} g_{r j}-\partial_{r} g_{s j}\right)=l^{-1} \varepsilon_{i j k} g_{s k}$, we find that the relation (47) becomes the electromagnetic Eulerian relations (17), (45) becomes (13), (46) becomes (22) and (23), (36) (with $\xi$ replaced by $x$ ) becomes (14), (49) becomes (12), (50) becomes (5), and (51) becomes (15).

The results of the previous section therefore provide us with the desired algorithm to compute the spinor field from the Lagrangian trajectories. Writing $\xi^{\mu}=\left(q_{i}, \theta_{r}\right)$ for the Lagrangian coordinates, the Lagrangian (31) becomes

$$
L=\int l \rho_{0}\left(q_{0}, \theta_{0}\right)\left(\left(A^{-1}\right)_{i r} \frac{\partial q_{i}}{\partial t} \frac{\partial \theta_{r}}{\partial t}-c^{2} A_{i r} \frac{1}{\rho^{2}} \frac{\partial \rho}{\partial q_{i}} \frac{\partial \rho}{\partial \theta_{r}}\right) \sin \theta_{01} d^{3} \theta_{0} d^{3} q_{0}
$$

Newton's law (35) reduces to the coupled relations

$$
\begin{aligned}
& \frac{\partial^{2} q_{i}}{\partial t^{2}}+\varepsilon_{i j k}\left(A^{-1}\right)_{k r} \frac{\partial q_{j}}{\partial t} \frac{\partial \theta_{r}}{\partial t}=-\frac{2 c}{\hbar} A_{i r} \frac{\partial Q}{\partial \theta_{r}} \\
& \frac{\partial^{2} \theta_{s}}{\partial t^{2}}+A_{s i} \frac{\partial}{\partial \theta_{r}}\left(A^{-1}\right)_{i q} \frac{\partial \theta_{q}}{\partial t} \frac{\partial \theta_{r}}{\partial t}=-\frac{2 c}{\hbar} A_{s i} \frac{\partial Q}{\partial q_{i}}
\end{aligned}
$$

where $A_{i r}$ is given by (21) with $\alpha_{r}$ replaced by $\theta_{r}\left(q_{0}, \theta_{0}, t\right)$ and we substitute

$$
\rho\left(q_{0}, \theta_{0}, t\right)=D^{-1}\left(q_{0}, \theta_{0}, t\right) \rho_{0}\left(q_{0}, \theta_{0}\right)
$$

into

$$
Q=-2 c \hbar A_{i r} \frac{1}{\sqrt{\rho}} \frac{\partial^{2} \sqrt{\rho}}{\partial \theta_{r} \partial q_{i}}
$$

with

$$
\frac{\partial}{\partial q_{i}}=J^{-1}\left(J_{i j} \frac{\partial}{\partial q_{0 j}}+J_{i s} \frac{\partial}{\partial \theta_{0 s}}\right), \quad \frac{\partial}{\partial \theta_{r}}=J^{-1}\left(J_{r j} \frac{\partial}{\partial q_{0 j}}+J_{r s} \frac{\partial}{\partial \theta_{0 s}}\right)
$$

Given the initial wavefunction $\psi_{0}(x, \alpha)=\chi_{0 a}(x) u_{a}(\alpha)=\sqrt{\rho_{0}} \exp \left(i S_{0} / \hbar\right)$ we can compute the wavefunction for all $x, \alpha, t$, up to a global phase, as follows. First, solve (54) and (55) subject to the initial conditions $\partial q_{0 i} / \partial t=(2 c / \hbar) A_{i r}\left(\theta_{0}\right) \partial S_{0} / \partial \theta_{0 r}$ and $\partial \theta_{0 r} / \partial t=(2 c / \hbar) A_{r i}\left(\theta_{0}\right) \partial S_{0} / \partial q_{0 i}$ to get the set of trajectories for all $q_{0}, \theta_{0}, t$. Next, invert these functions and substitute $q_{0}(x, \alpha, t)$ and $\theta_{0}(x, \alpha, t)$ in the right-hand side of (56) to find $\rho(x, \alpha, t)$ and in the right-hand sides of the equations 


$$
\partial_{r} S=\frac{\hbar}{2 c}\left(A^{-1}\right)_{i r} \frac{\partial q_{i}}{\partial t}, \quad \partial_{i} S=\frac{\hbar}{2 c}\left(A^{-1}\right)_{r i} \frac{\partial \theta_{r}}{\partial t}, \quad i, r=1,2,3
$$

to get $S$ up to an additive function of time, $\hbar f(t)$. To fix this function, apart from an additive constant, use (12). We obtain then the following formula for the wavefunction:

$$
\begin{aligned}
\psi(x, \alpha, t)= & \sqrt{\left(D^{-1} \rho_{0}\right)_{\substack{q_{0}(x, \alpha, t) \\
\theta_{0}(x, \alpha, t)}}} \\
& \times \exp \left[\frac{i}{2 c} \int\left(A^{-1}\right)_{i r} \partial q_{i} /\left.\partial t\right|_{\substack{q_{0}(x, \alpha, t) \\
\theta_{0}(x, \alpha, t)}} d \alpha_{r}+\left(A^{-1}\right)_{r i} \partial \theta_{r} /\left.\partial t\right|_{\substack{q_{0}(x, \alpha, t) \\
\theta_{0}(x, \alpha, t)}} d x_{i}+i f(t)\right]
\end{aligned}
$$

Finally, the components of the time-dependent spinor field may be read off from the formula (inverse of (6))

$$
\chi_{a}(x, t)=\int \psi(x, \alpha, t) u_{a}^{*}(\alpha) d \Omega
$$

Note that the trajectories depend on the basis set $u_{a}(\alpha)$ (which enter the calculation through the initial wavefunction) and would be different if a different choice to (7) were made. This will not affect the field values found from (61).

\section{HIDDEN-VARIABLE THEORY}

The theory of the Lagrangian paths is not in itself a hidden-variable theory, as that term was defined in the first sentence. It provides the groundwork for such a theory, however, and we need only take a simple further step. Thus, we suppose that a single one of the ensemble of paths associated with each set of Eulerian fields (i.e., $\chi$ ) is selected as special, or labelled, so that the outcome of a measurement is uniquely determined by this additional information together with $\chi$ (we assume that we are in a sufficiently low energy regime for this to be meaningful). The distribution of the hidden variables, i.e., the labels, is given by the position density $\rho=\chi^{+} \chi$. In the de Broglie-Bohm theory ${ }^{(6)}$ of massive systems it is postulated that a massive corpuscle occupies one of the paths and the hidden variable is its position, but of course this type of labelling is not available here. However the selection is made, the set of Lagrangian coordinates is just the complete set of hidden variables that may potentially be associated with the quantum system. Our deduction of the time variation of the wavefunction from the set of paths may therefore be regarded as evidence of the consistency of this hidden variable theory. Indeed, as argued elsewhere ${ }^{(3)}$, the Lagrangian coordinates constitute an alternative picture of quantum mechanics.

The paths are of the kind introduced in the de Broglie-Bohm theory but there are significant differences between that approach and the Lagrangian model ${ }^{(3)}$. A fundamental methodological distinction is that, while in both models the ensemble of trajectories generates $\rho$ given $\rho_{0}$, in the de Broglie-Bohm theory the orbits are found by first solving the Schrödinger equation. In the hydrodynamic model this procedure may be reversed and the orbits made the basis of the description.

It is noteworthy that the hydrodynamic paths do not form a Lorentz covariant structure. That we can derive the Lorentz covariant spin theory from a non-covariant model is not surprising. The absence of the fluid paths in the basic Eulerian equations suggests that $\rho(x)$ and $S(x)$ (and hence $\chi$ ) may be regarded as "collective coordinates" - functions that describe the bulk properties of the system without depending on the complex details of the particulate 
substructure. Features peculiar to the Eulerian picture, such as Lorentz covariance, may therefore be viewed as collective rather than fundamental properties. This result calls into question an assumption that pervades much of the discourse on hidden variables where it has generally been felt that a Lorentz covariant model is a necessary objective.

\section{REFERENCES}

1. F.J. Belinfante, A Survey of Hidden-Variables Theories (Pergamon Press, Oxford, 1973). 2. B. d'Espagnat, Conceptual Foundations of Quantum Mechanics, $2^{\text {nd }}$ edition (W.A. Benjamin, Reading MA, 1976).

3. P. Holland, Ann. Phys. (NY) 315, 503-529 (2005) quant-ph/0405145.

4. P. Holland, quant-ph/0411141.

5. I. Bialynicki-Birula, Acta Phys. Pol. B 26, 1201 (1995).

6. P.R. Holland, The Quantum Theory of Motion (Cambridge University Press, Cambridge, 2004).

7. E. Madelung, Z. Phys. 40, 322 (1926).

8. P. Holland, Ann. Phys. (Leipzig) 12, 446 (2003).

9. P. Holland and C. Philippidis, Phys. Rev. A 67, 062105 (2003).

10. P. Holland, in preparation.

11. J.P. Dahl, Kon. Danske Vid. Selsk. Mat-fys. Medd. 39, 12 (1977). 